\title{
ASOCIACIÓN ESTADÍSTICA ENTRE EL INGRESO Y LOS DERECHOS SOCIALES EN MÉXICO
}

\author{
Statistical association between income \\ and social entitlements in Mexico
}

Hada Melissa Sáenz Vela ${ }^{1}$, Luis Gutiérrez Flores² ${ }^{2}$ Enrique Eliseo Minor Campa ${ }^{3}$

Fecha de recepción: 18 de mayo de 2016

Fecha de aceptación: 21 de septiembre de 2016

1- Nacionalidad: Mexicana. Grado: Doctora en economía regional. Especialización: Economía regional. Adscripción: Universidad Autónoma de Coahuila. Correo: saenz.hm@gmail.com

2- Nacionalidad: Mexicana. Grado: Doctor en economía. Especialización: Economía regional. Adscripción: Universidad Autónoma de Coahuila. Correo: luis.gutierrez@uadec.edu.mx

3- Nacionalidad: Mexicana. Grado: Doctor en economía. Especialización: Pobreza. Adscripción: Coneval. Correo: eeminor@coneval.gob.mx 


\section{Resumen}

En México, el Consejo Nacional de Evaluación de la Política de Desarrollo Social estableció en su medición de la pobreza multidimensional que el ingreso y las carencias en materia de derechos sociales representan espacios independientes. No obstante, dicho supuesto no fue sometido a una verificación estadística, y su fundamentación es más bien teórica. Las técnicas estadísticas aquí propuestas, dado el carácter categórico de las variables involucradas, apuntan a la existencia de asociación entre ambos espacios. Los resultados obtenidos también sugieren omitir la carencia del acceso a los servicios de salud, y adicionalmente se encuentran relaciones entre las variables del espacio de los derechos, lo que conduce a problemas de redundancia entre las dimensiones.

Palabras clave: Pobreza multidimensional, ingreso, derechos sociales, tablas de contingencia.

\section{Abstract}

In Mexico, the National Council for the Evaluation of Social Development Policy established in its multidimensional measure of poverty, that income and gaps in social entitlements represent independent spaces. Nevertheless, such postulation was not submitted to statistical verification, and its basis is rather theoretical. The statistical techniques proposed in this work, given the categorical nature of the variables involved, lead to the existence of association between social entitlements and income. The results obtained also suggest omitting the gap in access to health services. Additionally, interesting relationships are found between the variables in the space of social entitlements, which lead to redundancy among dimensions.

Keywords: Multidimensional poverty, income, social entitlements, crosstabs. 


\section{Introducción}

$\mathrm{L}$

a medición de la pobreza desde un punto de vista multidimensional ha atraído a investigadores y tomadores de decisiones. No obstante que en la década de 1980 ya se hablaba de

la multidimensionalidad de la pobreza gracias a trabajos como el de Sen $(1983,1992)$, y su enfoque de capacidades y funcionamientos, fue hasta el año 2000 que el Banco Mundial reconoció finalmente la necesidad de medir multidimensionalmente los niveles de pobreza. Y es que diversos autores como Bourguignon y Chakravarty (2003) sostienen que el bienestar es intrínsecamente multidimensional desde el enfoque de capacidades de Sen, y que además es posible asociarlo también a formatos de medición como el de Necesidades Básicas Insatisfechas, un enfoque ampliamente utilizado en América Latina.

La literatura internacional ha señalado que el ingreso, como indicador del nivel de pobreza, puede resultar incompleto para conocer el verdadero nivel de privación que enfrentan los individuos. Alkire et al. (2015) en su capítulo introductorio reseñan diversas motivaciones por las cuales una perspectiva multidimensional puede aportar al entendimiento del fenómeno de la pobreza. Una razón recae en la ética y el reconocimiento de que la pobreza involucra juicios de valor. La otra cuestión es de origen empírico, ya que el ingreso puede ser insuficiente para capturar el nivel de privación de los individuos, además de los sesgos que provoca en la medición de tendencias de la pobreza. Y finalmente está la motivación política, es decir, informar para combatir la pobreza y mejorar el nivel de vida de la población.

A pesar del reconocimiento de la multidimensionalidad del fenómeno, que desde una perspectiva teórica ha sido ampliamente documentado, existe un conjunto de elementos metodológicos que deben de ser considerados. En primer lugar es necesario determinar las dimensiones, seguido de los umbrales bajo los cuales se establecerá que el individuo (u hogar) es carente. También habrá de elegirse la forma de ponderar cada dimensión, y el número de dimensiones carentes que debe presentar el individuo para ser considerado pobre multidimensional. ${ }^{4}$ Todos los temas anteriores se debaten aun en la literatura y en algunos elementos todavía no existe un consenso. La medida, ofrecida por Alkire y Foster (2011), es la que mayor impacto ha tenido en términos de su aplicación, ya que es fácilmente adaptable a diferentes contextos, además de contar con un esquema axiomático que la sustenta. ${ }^{5}$ En Sáenz (2016) se revisan algunos estudios recientes para analizar la forma en que se tomaron tales decisiones al aplicar la medida ; observando la coincidencia de sugerir la complementariedad necesaria entre las medidas monetarias y las multidimensionales.

En el caso de México, en 2004 se aprobó la Ley General de Desarrollo Social (LGDS). El Consejo Nacional de Evaluación de la Política de Desarrollo Social (CONEVAL) fue el organismo encargado de determinar la forma de identificar y medir la pobreza, de acuerdo a lineamientos y criterios establecidos por la misma Ley. La pobreza multidimensional en México tiene una definición que implica la ocurrencia de dos eventos simultáneos. Un individuo se clasifica como pobre multidimensional si su ingreso es menor a un mínimo establecido a través de las Líneas de Bienestar, y padece al menos una de seis

4- Se sugiere la revisión del capítulo 3 de Sáenz (2016).

5- Alkire et al. (2015) en el capítulo 5 muestran una amplia revisión de implementaciones de su medida . 
carencias (rezago educativo, acceso a servicios de salud, acceso a seguridad social, calidad y espacios de la vivienda, acceso a los servicios básicos de la vivienda y acceso a la alimentación).

Para el CONEVAL se tiene una combinación de dos enfoques: el de derechos sociales y el de bienestar. Respecto al primero, establece que todo individuo debe tener el acceso a un conjunto de garantías indispensables para la dignidad humana. Mientras que el segundo busca analizar el acceso individual a un conjunto de bienes y servicios, en términos del ingreso. Así, el Consejo establece que estos enfoques, a pesar de ser complementarios, son cualitativamente distintos y deben diferenciarse teórica y metodológicamente (CONEVAL 2009:27-29). Concluyendo que, bajo su concepción teórica, no es pertinente incluir el ingreso en la medida de intensidad de la pobreza multidimensional (ibíd.:46). Analizando la postura del CONEVAL es posible concluir que, además de la diferenciación teórica, es cierto que algunas políticas públicas podrían generar independencia de las carencias respecto del ingreso. Tal es el caso del acceso a los servicios de salud, esta variable considera el acceso al Seguro Popular, programa que ayudó a ampliar la cobertura sin necesidad de establecer un vínculo sólido con el nivel de ingresos del individuo. ${ }^{6}$

El objetivo del presente documento es explorar, de manera empírica, el comportamiento de la asociación existente entre el espacio de los derechos y el espacio del bienestar. Desde la teoría es comprensible la postura del Consejo, sin embargo, se invita a considerar las siguientes cuestiones. Metodologías como la de Alkire y Foster (2011) permiten conjuntar indicadores que conduzcan a una mejor medición de la pobreza; no sin antes realizar un análisis detallado de los indicadores que se pretende conformen la medida, aun cuando estos sean establecidos normativamente (Alkire et al., 2015). En función de lo anterior se sugiera que, dadas las observaciones realizadas por Alkire y Foster (2009) y Foster (2010) a la medida mexicana, se realice un estudio de las asociaciones entre las variables involucradas en la medición multidimensional. Es decir, la parte empírica del análisis permite corroborar que la medida a utilizar es lo más adecuada posible al contexto, en el caso de México, no se encontraron estudios previos que realicen un estudio similar al que aquí se desarrolla.

El documento consta de tres secciones principales. La primera aborda la revisión de literatura que refiere a la relación existente entre la pobreza de ingresos y la pobreza representada como índice de un conjunto de carencias de bienes o acceso a servicios básicos. Las Tablas de contingencia, los estadísticos, los estimadores y las medidas de asociación, que conforman una técnica adecuada al tipo de datos con los que se mide la pobreza en el país, se presentan en la segunda sección; es decir, permitirán explorar la relación entre el ingreso y las carencias de los derechos sociales. En el tercer apartado se muestran los resultados obtenidos del análisis de las variables involucradas en el estudio de la pobreza multidimensional en México. Se cierra con una sección de reflexiones finales.

\section{1. Índices de privación y su relación con el ingreso}

La conceptualización de la pobreza multidimensional recae en situaciones donde interesa la distribución conjunta de las dimensiones. Alkire et al. (2015) presentan múltiples técnicas desarrolladas en la literatura para realizar este tipo de exámenes. Sin embargo, cabe destacar una importante observación

6- Una descripción concisa del programa se encuentra en <http://www.salud.gob.mx/unidades/dgpfs/faq.htm>. 
que hacen los autores: si por cuestiones normativas algunos indicadores deben mantenerse en la medida, el análisis de redundancia y su tratamiento deben ser aclarados y justificados.

En el caso de México, no se realizó un análisis a las variables con las que medirían la pobreza. A pesar de existir observaciones previas, como la ofrecida por Foster (2010), donde se señalan redundancias entre las variables asociadas a los servicios de salud y a las de vivienda. El autor apunta a que dichas redundancias, de no ser corregidas conducen a una potencial doble ponderación de las dimensiones, lo cual se verá reflejado en los niveles de pobreza medidos. Además, Foster sugiere la utilización de la variable de ingreso junto a las carencias en la formulación de la medida multidimensional de pobreza. Esto es posible por las características de la medida, es decir, es una opción viable que se sustenta bajo el fundamento conceptual de la medición multidimensional. ${ }^{7}$

Esta sección tiene como objetivo mostrar estudios previos en la literatura internacional que encuentran asociación entre el nivel de ingreso y el nivel de pobreza de bienes (considerado como el acceso a un conjunto de bienes y servicios básicos). Si bien la asociación entre ambos espacios puede resultar intuitiva para el lector, este apartado pretende corroborar que empíricamente es posible determinarla a pesar de que la teoría sugiera lo contrario. Lo anterior invita también a plantearse que quizá algunas dimensiones de la pobreza siguen teniendo un gran peso y son interdependientes. ${ }^{8}$ Así, se ofrece un punto de partida para sugerir una revisión y justificación por parte del CONEVAL respecto del tratamiento que habrá de dar al supuesto de independencia entre el ingreso y la carencia de derechos sociales.

Un trabajo pionero es el de Ram (1982), donde se señalan las limitaciones al utilizar solamente al ingreso como medida de pobreza. No obstante, sugiere que medir separadamente cada una de sus diferentes dimensiones de la pobreza tampoco es recomendable. La técnica estadística utilizada por Ram es la de Análisis de Componentes Principales (ACP). Conforma cuatro índices: 1) conformado por grado de escolaridad, esperanza de vida y mortalidad infantil; 2) combina el índice anterior con el PIB per cápita; 3) se establece a partir de escolaridad, esperanza de vida, acceso a agua potable, número de personas por médico e ingesta de calorías; 4) combina el PIB per cápita con el tercer índice. Los datos corresponden a datos del periodo aproximado 1975-1978 para 147 países. Ofrece las correlaciones entre los índices compuestos y sus constituyentes; particularmente, en el tercer índice se encuentra un nivel considerable de asociación con el ingreso (0.82), el cual es ligeramente menor para el primer índice (0.64).

La propuesta de Nolan y Whelan (1996) ha sido ampliamente citada en la literatura. Ellos analizan la relación entre el ingreso y un conjunto de indicadores de privación a través de regresiones ordinarias. Con datos de 1987 para Irlanda se tomaron 24 preguntas sobre bienes y servicios considerados indispensables; todos ellos presentaron correlación negativa con el ingreso. Con la técnica de Análisis Factorial (AF) conforman tres índices (privación básica, privación secundaria y privación en el hogar) y uno más combinando todos los indicadores; los cuatro índices reportan niveles de correlación con el ingreso encontrándose entre 0.29 y 0.54 . Realizan regresiones que incluyen 15 variables explicativas para sus índices, incluyendo ingreso, características económicas, sociales y demográficas. Los resultados indican que el ingreso es significativo, además de la característica de contar con ahorros y contar con un historial de desempleo.

7- Se sugiere la lectura del documento de Alkire y Foster (2009), en el cual se sugiere al CONEVAL combinar al ingreso y los derechos sociales en la medida multidimensional.

8- El estudio de Battiston et al. (2013) señala que para Latinoamérica, el ingreso y la educación siguen siendo las dimensiones preponderantes de la pobreza. 
Para el caso de México es posible citar el trabajo de McKenzie (2005), quien mide desigualdad en lugar de pobreza con información del año 1998. Propone una medida formada por ACP y la compara contra otra donde usa la variable consumo en bienes no-durables (CND), generando cuatro medidas. Encuentra que a mayores niveles de CND y de ingreso corresponden mayores niveles de las medidas. El autor también calcula cinco índices de desigualdad, y en todos los casos se presenta una alta correlación entre los índices usando el CND y los obtenidos con la medida de ACP. Finalmente, con datos sobre migración para medir el efecto de la desigualdad sobre la asistencia a la escuela, encuentra que usar la medida ACP conduce a conclusiones similares a las obtenidas con el CND.

Un trabajo reciente es de Ucar (2015) para Turquía con datos del 2012 a partir de una encuesta con información sobre gasto en consumo, ingreso y un conjunto de bienes y servicios. Forma un índice de bienes a partir de 30 variables con ACP buscando analizar posibles traslapes entre quintiles ordenando por gasto, por ingreso y por índice. Sus resultados indican que el $54.1 \%$ y el $47.1 \%$ del quintil más bajo de acuerdo al índice también se ordenan como bajos en gasto y bajos en ingreso, respectivamente. Obtiene además el coeficiente de correlación de Pearson entre los quintiles, encontrando coeficientes de 0.75 entre gasto e ingreso, de 0.64 entre el índice y el gasto, y de 0.63 entre el índice y el ingreso. Respecto a los traslapes encuentra que el índice permite una mejor diferenciación entre los quintiles más bajos de la distribución.

En la siguiente sección se abordarán las técnicas estadísticas utilizadas en el análisis de datos categóricos. A pesar de ser medidas sencillas de calcular e interpretar, se encontraron pocos estudios dentro del análisis de los índices de privación donde se apliquen. Tal es el caso de Yu (2013) y Amores (2014) que recurren a algunas medidas de asociación. Sin embargo, al encontrar bajas asociaciones deciden no realizar modificación alguna a las dimensiones elegidas. En el caso de México, se propone dar robustez a la conclusión de asociación entre el ingreso y los derechos, así como a la de redundancia entre acceso a servicios de salud y acceso a la seguridad social; por lo tanto se explorarán diversas medidas estadísticas que fundamenten los resultados.

\section{Propuesta metodológica: Tablas de contingencia y medidas de asociacion}

Cuando se tienen datos de tipo categórico, una manera de presentar la relación entre las variables es a través de tablas de contingencia. Sean las variables categóricas $X$ y $Y$, con $I$ y J niveles, respectivamente. Si se divide la frecuencia reportada en cada celda $\left(n_{\mathrm{ij}}\right)$ entre el tamaño de la muestra $(n)$ se obtienen las probabilidades de que una respuesta pertenezca a la categoría $(i, j)$, de manera que $\pi_{\mathrm{ij}}=n_{\mathrm{ij}} / n$. Sea $\pi_{i j}=P\left(x_{\mathrm{i}}, y_{\mathrm{j}}\right)$ la distribución de probabilidad conjunta de $(\mathrm{X}, \mathrm{Y})$, esto es, la probabilidad de que la respuesta $\left(x_{i}, y_{\mathrm{j}}\right)$ ocurra en la celda $(i, j)$ con $i=1: I$ y $j=1: j$; mientras que las distribuciones marginales están dadas por los totales por fila y columna, denotadas por $\left\{\pi_{i+}\right\}$ para la suma por fila y $\left\{\pi_{+j}\right\}$ para la suma por columna, cumpliéndos

$$
\begin{aligned}
& \pi_{i+}=\sum_{j i j} \mathrm{y} \pi_{+j}=\sum_{i \pi_{i j}}, \\
& \sum_{i \pi_{i+}}=\sum_{j} \pi_{+j}=\sum_{i} \sum_{j} \pi_{i j}=1 .
\end{aligned}
$$


Por lo anterior, en el Cuadro 1 se muestra el formato general de la notación de las probabilidades conjunta, condicional y marginal para una tabla $I x J .{ }^{9}$

\section{Cuadro 1. Notación para probabilidades conjunta, condicional y marginal de una tabla $I x J$.}

\begin{tabular}{|c|c|c|c|c|c|}
\hline & \multicolumn{4}{|c|}{ Y } & \multirow[b]{2}{*}{ Total } \\
\hline$X$ & Col. 1 & Col. 2 & $\ldots$ & Col. & \\
\hline Fila 1 & $\left(\begin{array}{c}\pi_{11} \\
\left(\pi_{1 \mid 1}\right)\end{array}\right.$ & $\left(\begin{array}{c}\pi_{12} \\
\left(\pi_{2 \mid 1}\right)\end{array}\right.$ & ... & $\begin{array}{c}\pi_{11} \\
\left(\pi_{1 \mid 1}\right)\end{array}$ & $\begin{array}{l}\pi_{1+} \\
\text { (1) }\end{array}$ \\
\hline Fila 2 & $\left(\begin{array}{c}\pi_{21} \\
\left(\pi_{1 \mid 2}\right)\end{array}\right.$ & $\left(\begin{array}{c}\pi_{22} \\
\left(\pi_{2 \mid 2}\right)\end{array}\right.$ & ... & $\begin{array}{c}\pi_{21} \\
\left(\pi_{|| 2}\right)\end{array}$ & $\begin{array}{l}\pi_{2+} \\
(1)\end{array}$ \\
\hline$\vdots$ & $\vdots$ & $\vdots$ & $\because$ & $\vdots$ & $\vdots$ \\
\hline Fila & $\left(\begin{array}{c}\pi_{11} \\
\left(\pi_{1||}\right)\end{array}\right.$ & $\begin{array}{l}\pi \_12 \\
\left(\pi_{2||}\right)\end{array}$ & ... & $\begin{array}{l}\pi_{-} \mid \boldsymbol{J} \\
\left(\pi_{\|\|}\right)\end{array}$ & $\begin{array}{l}\pi_{1+} \\
(1)\end{array}$ \\
\hline Total & $\pi_{+1}$ & $\pi_{+2}$ & ... & $\pi_{+1}$ & (1) \\
\hline
\end{tabular}

Fuente: Adaptación de Agresti (2002:49).

Dado un sujeto clasificado en la fila $i$ de $X, \pi_{j \mid i}$ denota la probabilidad de clasificarlo en la columna $j$ de $\mathrm{Y}$, de manera que $\sum_{j} \pi_{j \mid i}=1$. El conjunto $\left\{\pi_{i \mid i} \pi_{2 \mid i}, \ldots, \pi_{\mathrm{Jj}}\right\}$ forma la distribución condicional de $Y$ dada la categoría $i$ de $X$. La distribución condicional de $Y$ dado $X$ se relaciona con la conjunta de la forma $\pi_{j \mid i}=\pi_{i j} / \pi i+, \forall i, j$. Dos variables categóricas se definen como independientes si todas las probabilidades conjuntas son iguales al producto de sus probabilidades marginales; es decir, $\pi_{i j}=\pi_{i+} \pi_{+j}$, $\forall i^{\prime} j$. Si X y Y son independientes se cumple que $\pi_{j \mid i}=\pi_{+j}, \forall_{i}$. Dicho de otra forma, dos variables son independientes si las proporciones por fila de una misma columna son las mismas y esto se cumple para todas las columnas.

\subsection{Test $x^{2}$ de Pearson}

Si las distribuciones condicionales de $\mathrm{X}$ sobre los diferentes niveles de $\mathrm{Y}$ son idénticas; es decir, si $\left\{\pi_{j \mid 1}=\pi_{j \mid 2}=\cdots=\pi_{j \mid p} ; j=1: J\right\}$, entonces se dicen independientes. Pero esto es difícil conseguirlo dada una muestra, una forma de verificar si estas variaciones se deben solamente al muestreo es mediante la prueba $x^{2}$ de Pearson.

La hipótesis nula es que las variables son estadísticamente independientes. Se comparan las frecuencias esperadas $f_{e}$ bajo la hipótesis de independencia contra las observadas $f_{o}$. Los valores $f_{e}$ son iguales al 
producto de los totales por fila y columna de esa celda, dividido por el tamaño muestral total. De manera tal que, el estadístico se escribe como:

$$
\chi^{2}=\sum \frac{\left(f_{o}-f_{e}\right)^{2}}{f_{e}},
$$

donde la suma se hace sobre todas las celdas de la tabla de contingencia. Si la independencia se cumple, los valores $f_{o}$ y $f_{e}$ serán cercanos, y por tanto el valor de $x^{2}$ será relativamente pequeño. Cuanto mayor el $x^{2}$, mayor la evidencia contra la hipótesis nula; aunque esto no da información sobre la fuerza de la asociación entre las variables. La ventaja de la prueba $x^{2}$ recae en el tratamiento que da a las variables. Esto porque el estadístico no depende de cuál variable ha sido elegida como respuesta, de hecho, los resultados son idénticos ante cualquier elección.

\subsection{Razón de posibilidades}

Para determinar qué tan asociadas se encuentran dos variables se puede recurrir al concepto de razón de posibilidades. ${ }^{10}$ Siguiendo la notación de la Tabla 1 se pueden formar sub-tablas $2 \times 2$, como la mostrada en el Cuadro 2.

\section{Cuadro 2. Sub-tabla 2x2 que puede formarse de una IxJ.}

\begin{tabular}{|c|c|c|c|}
\hline & \multicolumn{2}{|c|}{$Y$} & \\
\hline $\mathrm{X}$ & Col. 1 & Col. 2 & Total \\
\hline Fila 1 & $\begin{array}{r}\pi_{11} \\
\left(\pi_{1 \mid 1}\right)\end{array}$ & $\begin{array}{c}\pi_{12} \\
\left(\pi_{2 \mid 1}\right)\end{array}$ & $\begin{array}{c}\pi_{1+} \\
(1)\end{array}$ \\
\hline Fila 2 & $\begin{array}{c}\pi_{21} \\
\left(\pi_{1 \mid 2}\right.\end{array}$ & $\begin{array}{c}\pi_{22} \\
\left(\pi_{2 \mid 2}\right)\end{array}$ & $\pi_{2+}$ \\
\hline Total & $\pi_{+1}$ & $\pi_{+2}$ & $(1)$ \\
\hline
\end{tabular}

Fuente: Adaptación de Agresti (2002).

Para los sujetos en la fila $i, \pi_{1 \mid \mathrm{i}}$ es la probabilidad de que la respuesta tenga un resultado "éxito" en la categoría 1 de $X$. Con solamente dos posibles resultados, se tiene que $\pi_{2 \mid i}=1-\pi_{1 \mid i}$ por lo que la notación se simplifica a $\pi_{\mathrm{i}}$ en lugar de $\pi_{1 \mid \mathrm{i}}$. La diferencia de proporciones de éxitos $\pi_{1}-\pi_{2}$, es la comparación básica de dos filas; la comparación de la categoría 2 (o "fracaso" de $X$ ) se calcula como $\left(1-\pi_{1}\right)-\left(1-\pi_{2}\right)=\pi_{2}-\pi_{1}$. La diferencia de proporciones caerá entre -1 y +1 ; si las distribuciones condicionales son iguales, las diferencias serán cero. La variable respuesta $Y$ es estadísticamente independiente de la clasificación de las filas cuando se cumple $\pi_{1}-\pi_{2}=0$.

10- En inglés es llamado Odds Ratio, sin embargo, este término no tiene una traducción al español aceptada. Aquí se referirá a estos términos como posibilidades y razón de posibilidades, una discusión más amplia al respecto se encuentra en Tapia y Nieto (1993) y en Martí et al. (2006). 
La posibilidad (odds) de éxito se define a partir de la probabilidad de éxito en la variable $X$. En el contexto de las tablas de contingencia, sea $\pi_{\mathrm{i}}$ la probabilidad de éxito, entonces la posibilidad se escribe como:

$$
\Omega_{i}=\frac{\pi_{i}}{1-\pi_{i}}
$$

Si $\Omega_{i}>1$, la posibilidad de éxito es mayor que la de fracaso. Así, la razón de posibilidades se escribe como

$$
\theta=\frac{\Omega_{1}}{\Omega_{2}}=\frac{\pi_{1} /\left(1-\pi_{1}\right)}{\pi_{2} /\left(1-\pi_{2}\right)} \text {. }
$$

La razón de posibilidades, $\theta$, puede tomar cualquier valor no negativo. Particularmente, cuando $\theta=1$ significa que las variables $X$ y $Y$ son independientes; por otro lado, $\theta>1$ indica que es más probable que los sujetos de la fila 1 tengan un éxito que los sujetos de la fila 2. Por tanto, valores altos de $\theta$ representan una mayor asociación entre las variables (Agresti, 2002). Es importante señalar que $\theta$ no cambia su valor cuando se intercambia la orientación de la tabla, por lo que no es necesario identificar a una de las variables como variable de respuesta. Cuando se tienen tablas $I x J$, las razones de posibilidades describirán los patrones por sub-tablas $2 \times 2$.

Para la inferencia, la independencia corresponde a $H_{o}: \log _{\theta}$. El logaritmo de la razón de posibilidades es simétrico sobre su valor, intercambiar filas por columnas resultará en un cambio de signo. La significación de estos $\log \theta$ se puede obtener con el test $z$, el cual se calcula como

$$
Z=\frac{\text { Valor estimado-Valor de la hipótesis nula }}{\text { Error estándar }} .
$$

\subsection{Medidas de asociación y correlación}

Para medir el grado de asociación entre dos variables existen otras alternativas, una opción es recurrir a pruebas no paramétricas. A continuación se presentan algunas medidas usuales que se emplean al hacer análisis de variables categóricas.

\subsubsection{Indices basados en $x^{2}$}

Sea $n$ el total de elementos en la tabla, $y m=\min$ \{número de filas, número de columnas\}. Se definen los siguientes coeficientes, los cuales son medidas que intentan que el estadístico $x^{2}$ tome un valor entre $0 \mathrm{y}$ 1, además de informar el grado de asociación existente:

$$
\begin{aligned}
& \text { Coeficiente } \phi=\sqrt{\frac{\chi^{2}}{n}}, \quad(8) \\
& \text { Coeficiente de contingencia } C=\sqrt{\frac{\chi^{2}}{\chi^{2}+n}},
\end{aligned}
$$




\section{Coeficiente $V$ de Cramer $V=\sqrt{\frac{\chi^{2}}{n(m-1)}}(10)$}

De Visauta (2007) se tienen algunas observaciones sobre estas medidas. En tablas 2x2, el valor de $\phi$ y $V$ es idéntico al coeficiente de correlación de Pearson. Si el número de filas o de columnas es superior a 2, entonces $\phi$ puede ser superior a la unidad. El problema con el índice $V$ de Cramer es que tiende a subestimar el grado de asociación entre las variables.

\subsubsection{Medidas ordinales}

Cuando existe ordenación en las categorías de los datos, se recurre a las medidas $\gamma, \tau_{b} \tau_{c} D$ de Somers y $\rho$ de Spearman. Las variables ordinales comúnmente presentan tendencias de asociación, esto es, respuestas $Y$ tienden a crecer si $X$ lo hace, $o$ a reducirse si $X$ lo hace.

Se dice que un par de datos es concordante si el individuo presenta un alto valor en $X$ y también en $Y$; se dicen discordantes cuando un alto valor de $X$ se asocia con uno bajo de $Y$. Sean $\Pi_{c}$ la suma de los pares concordantes, $\Pi_{d}$ la suma de los pares discordantes, $n_{p}$ el número total de pares, $m$ el min $\{$ número de filas, número de columnas\}, $\mathrm{T}_{X}$ el número de pares empatados en $X, \mathrm{y} T_{Y}$ el número de pares empatados en $Y$ (son empatados si dos casos tienen valores idénticos en una o en las dos variables). Se definen entonces las siguientes medidas:

$$
\begin{aligned}
\gamma & =\frac{\Pi_{c}-\Pi_{d}}{\Pi_{c}+\Pi_{d}}, \\
\tau_{b} & =\frac{\Pi_{c}-\Pi_{d}}{\sqrt{\left(\Pi_{c}+\Pi_{d}+T_{X}\right)\left(\Pi_{c}+\Pi_{d}+T_{Y}\right)}} \\
\tau_{c} & =\frac{2 m\left(\Pi_{c} \Pi_{d}\right)}{n^{2}(m-1)}
\end{aligned}
$$

Estas tres medidas informan sobre la dirección de la asociación de las variables.

Explica Visauta (2007) que, dado que los empates pueden darse en una variable u otra, o en ambas, la $\tau_{\mathrm{b}}$ refleja los empates que no sean en ambas variables; y la $\tau_{c}$ aproxima el valor a la escala $+10-1$ para cualquier tabla $I x J$. La medida $\gamma$ trata a la variable de manera simétrica, variando entre $-1 \mathrm{y}+1$. Si las variables son independientes, entonces $\gamma=0$; pero un $\gamma=0$ no necesariamente significa que haya independencia. El Cuadro 3 puede funcionar como una guía sobre los valores absolutos de las medidas de asociación $V$ de Cramer, y las $\tau_{b} \mathrm{y} \tau_{c}{ }^{11}$

11- Botsch (2011) señala que sus notas son resultado de su propia experiencia, pero son útiles como una primera guía de resultados. 


\begin{tabular}{|c|c|}
\hline Valor & Nivel de asociación \\
\hline Menor a 0.10 & Muy débil \\
\hline De 0.10 a 0.19 & Débil \\
\hline De 0.20 a 0.29 & Moderada \\
\hline Mayor 0.30 & Fuerte \\
\hline
\end{tabular}

Fuente: Adaptación de Botsch (2011).

En la $D$ de Somers, se considera a una variable como dependiente y a la otra como independiente. El valor absoluto indica la fortaleza de la asociación (variando entre -1 y 1), el signo indica si es positiva o negativa, y si es cero entonces las variables se dicen independientes. Así, se tienen tres versiones para la $D$ de Somers:

$$
\begin{aligned}
& \text { Simétrica, } D=\frac{\Pi_{c}-\Pi_{d}}{\Pi_{c}+\Pi_{d}+\left(\frac{T_{X}+T_{Y}}{2}\right)}, \\
& X \text { dependiente, } D_{X}=\frac{\Pi_{c}-\Pi_{d}}{\Pi_{c}+\Pi_{d}+T_{X}}, \\
& Y \text { dependiente, } D_{Y}=\frac{\Pi_{c}-\Pi_{d}}{\Pi_{c}+\Pi_{d}+T_{Y}}
\end{aligned}
$$

Si los datos no cumplen el supuesto de normalidad, resulta apropiado calcular el coeficiente de correlación $\rho_{S}$ de Spearman. Nuevamente el valor absoluto representa la fuerza de asociación, y el signo la dirección; su ventaja radica en la robustez ante valores extremos. Para obtenerlo, primero se ordenan los datos de $X$ y $Y$ y se reemplazan por su respectivo orden; sea $d$ la diferencia entre los correspondientes estadísticos de orden de $\mathrm{x}_{i}-\mathrm{y}_{i}$ por lo que se define como

$$
\rho_{S}=1-\frac{6 \sum_{i} d_{i}^{2}}{n\left(n^{2}-1\right)}
$$

\subsubsection{Correlación entre variables categóricas}

Cuando se tienen variables de tipo categórico no es recomendable utilizar la correlación de Pearson. Para dichas situaciones se tienen fórmulas de correlación que consideran ese tipo de situaciones: poli- 
serial y biserial cuando una variable es continua y la otra categórica; tetracórica si ambas variables son dicotómicas; y policórica si alguna categórica es ordinal.

Estas correlaciones siguen un comportamiento similar al del tradicional coeficiente de correlación de Pearson. Señala Sheskin (2004) que la correlación tetracórica y la biserial también cumplen encontrarse en el rango [-1,1], y si la relación entre las variables es no lineal, el valor calculado solo representará el grado de relación lineal entre las variables. De igual forma las versiones poliserial y policórica tienen interpretaciones similares, ya que la diferencia recae en las formulaciones para obtener la correlación y los métodos con los que se aproximan cuando las variables son de tipo ordinal en lugar de binaria. ${ }^{12}$

\section{Resultados para México}

En México, de acuerdo con CONEVAL, un individuo se define como pobre multidimensional si es carente tanto en el espacio de los ingresos como en el de los derechos sociales. El indicador que se asocia al espacio de los ingresos es el ingreso corriente total per cápita. El espacio de los derechos se define a través de seis indicadores de carencias: rezago educativo, acceso a los servicios de salud, acceso a la seguridad social, calidad y espacios de la vivienda, servicios básicos de la vivienda y acceso a la alimentación.

Se presenta carencia en el primer espacio cuando un individuo no cuenta con el ingreso suficiente para adquirir los bienes y servicios básicos requeridos. Estos bienes y servicios son determinados por el CONEVAL, a lo cuales se les asocia un costo que definirá a la denominada Línea de Bienestar (LB). En el segundo espacio alguien es carente cuando al menos uno de sus derechos se ve limitado; de esta manera, se construye el Î́ndice de Privación Social (IPS) como una combinación lineal unitaria de los seis indicadores asociados. Por lo tanto, un individuo es carente de derechos sociales cuando su IPS es mayor o igual a uno.

En Sáenz et al. (2015) se presenta un análisis de la medida mexicana de pobreza multidimensional. El estudio describe los elementos que conforman la medida de Alkire y Foster (2011), para luego verificar que la medida mexicana, llamada IPM, es una adaptación de la primera. Se detallan los elementos que diferencian a la IPM, cuáles son sus limitaciones en términos de interpretación, y se realiza una primera exploración sobre la asociación entre los elementos que conforman la medición. El IPM deja fuera del análisis a los no-pobres de ingresos pero que cuentan con carencias (lo cual reporta en otra medida, CONEVAL 2009:46) a pesar de que la permitiría combinar ambos espacios. Mientras que las razones de posibilidades señalan mayor asociación del ingreso con ciertas carencias de derechos, y un importante nivel de redundancia entre las referentes a los servicios de salud.

En el Cuadro 4 se presentan los nombres de las variables a emplear en el resto del documento, además de especificar el tipo de variable. Estos datos se obtuvieron de la base brindada por CONEVAL del MCS de la ENIGH 2012 en su página oficial para el cálculo de la pobreza multidimensional (excepto ing.bc e ips.sal). ${ }^{13}$ Si bien el ingreso es el mismo para todos los integrantes de un hogar, cada uno puede presentar un diferente nivel de carencias, es por ello que en México el estudio de la pobreza multidimensional se

12- Se sugiere la revisión de documentos como Kirk (1973) y Olsson et al.(1982), los cuales son pioneros en las formulaciones y aproximaciones para obtener los coeficientes de correlación para variables categóricas.

13- Al final del documento encontrará un anexo donde se describen las carencias de derechos sociales. 
realice a nivel individual. En adelante, se hablará de carencias para referirse solamente a la condición de carencias en el espacio de los derechos sociales.

Cuadro 4.Variables consideradas en la medición de la pobreza multidimensional.

\begin{tabular}{|c|c|}
\hline Variable & Tipo \\
\hline ic_rezedu & Dicotómica. Presenta la carencia de rezago educativo. \\
\hline ic_asalud & Dicotómica. Presenta la carencia de acceso a servicios de salud. \\
\hline ic_segsoc & Dicotómica. Presenta la carencia de acceso a seguridad social. \\
\hline ic_cv & Dicotómica. Presenta la carencia en la calidad y espacios de la vivienda. \\
\hline ic_sbv & Dicotómica. Presenta la carencia en los servicios básicos de la vivienda. \\
\hline ic_ali & Dicotómica. Presenta la carencia en el acceso a la alimentación. \\
\hline ips & Ordinal, de 0 a 6. Índice de Privación Social. \\
\hline ips.sal & Ordinal, de 0 a 5, se elimina la carencia ic_asalud del IPS. \\
\hline ictpc & Continua. Ingreso corriente total per cápita. \\
\hline ing.bc & Continua. Transformación Box-Cox de ictpc. \\
\hline plb & Dicotómica. Pobre de ingresos. \\
\hline pobreza & Dicotómica. Pobre multidimensional. \\
\hline
\end{tabular}

Fuente: Elaboración propia a partir de información de CONEVAL.

La variable del ingreso también es de interés en el presente estudio, a pesar de no figurar directamente en la definición de pobreza multidimensional. El CONEVAL define al ingreso corriente total como la suma del ingreso corriente monetario y no-monetario del hogar, el cual luego se ajusta por economías de escala y escalas de adulto equivalente cuando el tamaño del hogar es mayor a uno, para formar el ingreso per cápita, ictpc. ${ }^{14}$ Se añadió una transformación Box-Cox de ictpc debido a que esta variable presenta algunos inconvenientes. ${ }^{15}$

En la muestra con ingresos acotados hay un total de 210 '950 observaciones, de las cuales $47.3 \%$ son pobres multidimensionales y $52.7 \%$ son pobres de ingresos; tal como lo señala el Cuadro 5 . De las frecuencias de los valores del IPS, observe que el 50.3\% de la muestra padece una o dos carencias. La caren-

14- El primero considera remuneraciones por trabajo subordinado, ingreso por trabajo independiente (incluye autoconsumo), ingreso por renta de la propiedad, otros ingresos provenientes del trabajo y transferencias. Mientras que el ingreso corriente no-monetario comprende los pagos en especie, transferencias en especie (regalos en especie, se excluyen las transferencias que se dan por única vez) y no incluye renta imputada.

15- En primer lugar porque la muestra del MCS cuenta con 870 reportes de cero ingresos y 674 de ingreso menor a 50 pesos mensuales. En segundo lugar, en cuanto a ingresos altos, hay 180 reportes al considerar ingresos mayores a 50 '000 pesos mensuales, siendo el mayor de $508^{\prime} 700$ pesos. Dado lo anterior y tras realizar varias pruebas, se decidió utilizar únicamente la información referente a los individuos que contaran con un ingreso mensual entre 50 y 50'000 pesos. Con esto se intenta reducir el exceso de valores atípicos que pudieran sesgar los resultados. Los datos eliminados representan menos del $1 \%$ de la muestra total y el $1.30 \%$ de los pobres multidimensionales, lo que no implica una reducción significativa de la muestra. 
cia más padecida por la población es la de acceso a seguridad social seguida del acceso a la alimentación; la menos padecida es la de condiciones y espacios de la vivienda.

Cuadro 5. Frecuencias de las variables utilizadas.

\begin{tabular}{|c|c|c|c|}
\hline Variable & \multicolumn{2}{|c|}{ Frec. Absolutas } & Frec. Relativas (\%) \\
\hline \multirow{2}{*}{ pobreza } & 0 & $112^{\prime} 269$ & 53.2 \\
\hline & 1 & $98^{\prime} 681$ & 46.8 \\
\hline \multirow{7}{*}{ ips } & 0 & $53^{\prime} 230$ & 25.2 \\
\hline & 1 & $52 ' 888$ & 25.1 \\
\hline & 2 & $52^{\prime} 731$ & 25 \\
\hline & 3 & $30 ’ 856$ & 14.6 \\
\hline & 4 & $15^{\prime} 171$ & 7.2 \\
\hline & 5 & $5^{\prime} 120$ & 2.4 \\
\hline & 6 & 954 & 0.5 \\
\hline
\end{tabular}

Fuente: Elaboración propia en R a partir de MCS-ENIGH 2012.

Otro elemento de interés son las correlaciones entre variables, las cuales se presentan en el Cuadro 6 , donde todas las correlaciones resultaron significativas al 99\% de confianza. Existe una correlación negativa entre el ingreso, más acentuado al considerar el ingreso transformado, y el Índice de Privación Social, siendo de -0.370 y -0.519 , respectivamente. De igual manera, ante un incremento en el ingreso se presenta una reducción en la probabilidad de padecer las carencias, el mayor valor se da entre el ingreso y la seguridad social (-0.491). La variable pobreza de ingresos, plb, también presenta niveles moderados de correlación, siendo la más alta con el acceso a la seguridad social (0.471) y la menor con el acceso a los servicios de salud (0.101). Precisamente, el acceso a servicios de salud y a la seguridad social son las variables con mayor correlación entre sí (0.731), considerando la baja asociación reportada por el acceso a servicios de salud con las demás carencias. Se observa también una moderada asociación entre las carencias asociadas a la vivienda, alcanzando 0.547 .

Cuadro 6. Matriz de correlaciones entre las variables de estudio.

\begin{tabular}{|c|c|c|c|c|c|c|c|c|c|c|c|}
\hline Variables & ing.bc & ictpc & plb & ips & ips.sal & ic_rezedu & $\begin{array}{c}\text { ic_asa- } \\
\text { lud }\end{array}$ & $\begin{array}{c}\text { ic_- } \\
\text { segsoc }\end{array}$ & ic_cv & ic_sbv & ic_ali \\
\hline ing.bc & 1 & 0.799 & -0.946 & -0.519 & -0.556 & -0.291 & -0.098 & -0.491 & -0.411 & -0.531 & -0.377 \\
\hline ictpc & & 1 & -0.679 & -0.37 & -0.396 & -0.223 & -0.073 & -0.361 & -0.275 & -0.339 & -0.29 \\
\hline plb & & & 1 & 0.474 & 0.509 & 0.262 & 0.101 & 0.471 & 0.414 & 0.433 & 0.399 \\
\hline ips & & & & 1 & 0.908 & 0.546 & 0.588 & 0.749 & 0.666 & 0.71 & 0.598 \\
\hline
\end{tabular}




\begin{tabular}{|c|c|c|c|c|c|c|c|c|c|c|c|}
\hline Variables & ing.bc & ictpc & plb & ips & ips.sal & ic_rezedu & $\begin{array}{l}\text { ic_asa- } \\
\text { lud }\end{array}$ & $\begin{array}{c}\text { ic } \\
\text { segsoc }\end{array}$ & ic_cv & ic_sbv & ic_ali \\
\hline ips.sal & & & & & 1 & 0.587 & 0.286 & 0.707 & 0.714 & 0.77 & 0.654 \\
\hline ic_rezedu & & & & & & 1 & 0.099 & 0.132 & 0.24 & 0.358 & 0.196 \\
\hline ic_asalud & & & & & & & 1 & 0.731 & 0.086 & 0.056 & 0.051 \\
\hline ic_segsoc & & & & & & & & 1 & 0.377 & 0.482 & 0.285 \\
\hline ic_cv & & & & & & & & & 1 & 0.547 & 0.364 \\
\hline ic_sbv & & & & & & & & & & 1 & 0.348 \\
\hline ic_ali & & & & & & & & & & & 1 \\
\hline
\end{tabular}

Fuente: Elaboración propia en R a partir de MCS-ENIGH 2012.

En esta primer etapa se analiza la asociación entre la condición de pobreza de ingresos (plb) contra la condición de ser carente en cada uno de los derechos sociales. Se utilizaron tablas de contingencia $2 \times 2$, para las combinaciones pobreza por ingreso-carencia. Fue posible aplicar el Test $\chi^{2}$ de Pearson, donde se probó la hipótesis de independencia entre dos variables, la cual se rechazó en todos los casos con un p-valor igual 0 .

Cuadro 7. Medidas de asociación entre plb y carencias.

\begin{tabular}{|c|c|c|c|c|c|c|c|c|}
\hline & \multicolumn{7}{|c|}{ Medidas de asociación } \\
\hline Carencia & $\mathrm{C}$ & $\mathrm{V}$ & $\gamma$ & $\tau_{b}$ & $\tau_{c}$ & $D$ & $\rho_{s}$ & $\theta$ \\
\hline ic_rezedu & 0.144 & 0.146 & 0.361 & 0.146 & 0.117 & 0.182 & 0.146 & 2.13 \\
\hline ic_asalud & 0.056 & 0.056 & 0.142 & 0.056 & 0.044 & 0.071 & 0.056 & 1.33 \\
\hline ic_segsoc & 0.294 & 0.308 & 0.574 & 0.308 & 0.300 & 0.316 & 0.308 & 3.69 \\
\hline ic_cv & 0.203 & 0.207 & 0.586 & 0.207 & 0.143 & 0.298 & 0.207 & 3.83 \\
\hline ic_sbv & 0.243 & 0.250 & 0.568 & 0.250 & 0.211 & 0.296 & 0.250 & 3.63 \\
\hline ic_ali & 0.228 & 0.234 & 0.523 & 0.234 & 0.201 & 0.271 & 0.234 & 3.19 \\
\hline
\end{tabular}

Nota: El valor de la D de Somers que se reporta es el valor más alto, el cual ocurre cuando plb es la variable dependiente. Se omiten los valores de puesto que en tablas $2 \times 2$ son idénticos a los ofrecidos por la $\mathrm{V}$ de Cramer.

Fuente: Elaboración propia en R a partir de MCS-ENIGH 2012.

Para determinar el grado y dirección de asociación entre la pobreza por ingresos y cada carencia se calcularon diferentes medidas, cuyos resultados se muestran en el Cuadro 7. Las medidas $\theta$ se leen como la posibilidad de que un no-pobre sea no-carente es $\theta$ veces la posibilidad de que un pobre sea no-carente. Así, puede verse que para todas las carencias los individuos no-pobres tienen una mayor posibilidad de ser no-carentes que los pobres, de manera que ser pobre de ingresos se relaciona con presentar carencias. El acceso a la salud tiene un $\theta$ cercano a uno, pero aun así es significativamente distinto (de acuerdo a la 
prueba $z$ que fue aplicada a los seis parámetros). Los $\theta$ y su versión logarítmica indican que las variables con menor asociación con la pobreza de ingresos son la carencia de servicios de salud y el rezago educativo.

Las medidas $C$ y $V$ señalan la existencia de una moderada asociación entre la pobreza de ingresos y las carencias. Para el acceso a servicios de salud se reporta un nivel muy bajo (0.056), mientras que el mayor se da en acceso a seguridad social (0.308), para cualquiera de estas dos medidas. Por otro lado, las medidas direccionales $\left(\gamma, \tau_{b}, \tau_{c}, D\right.$ y $\left.\rho_{s}\right)$ indican que la pobreza de ingresos se asocia positivamente con la presencia de las carencias, incluso para el acceso a los servicios de salud. El orden de menor a mayor asociación varían ligeramente en las primeras 7 medidas; mientras que los valores de $\theta$ reportan una ordenación distinta, pero las de mayor valor siguen siendo las variables de servicios básicos en la vivienda, condiciones de la vivienda y acceso a seguridad social.

De estos resultados es importante destacar algunos elementos. La carencia de servicios de salud es la que menor asociación tiene con la pobreza de ingresos (para las ocho medidas). Es posible que la baja asociación se deba a las múltiples formas de poder acceder a este derecho y la facilidad de acceder al Seguro Popular, pues el monto para inscribirse en el servicio es muy bajo y se define en función de nivel de ingreso de los individuos. Respecto de las carencias relacionadas con la vivienda, se nota una menor asociación en las condiciones de la vivienda (ic_cv). Esto último puede deberse a los esfuerzos de los gobiernos federal y estatales de reducir el número de viviendas con piso de tierra, o con los programas para combatir el hacinamiento. La variable de servicios básicos en la vivienda (ic_sbv) no tiene que ver solamente con la mayor cobertura de los servicios de agua, drenaje y electricidad, sino con la capacidad de los individuos de gozar de ellos, por ello que probablemente registre una asociación mayor con el ingreso. Por otro lado, la asociación más alta se da en la variable del acceso a seguridad social (ic_segsoc), de acuerdo a las siete primeras medidas de la Tabla 7. En esta variable se considera a la población que cuenta con beneficios a partir del empleo formal, en este sentido, se espera que el empleo formal garantice un nivel de ingresos es capaz de proporcionar un conjunto mínimo de bienes y servicios requeridos para una vida digna. Por lo tanto, no contar con un empleo formal implicará una mayor incidencia de pobreza de ingresos.

Cuadro 8. Razones de posibilidad entre las carencias.

\begin{tabular}{|c|c|c|c|}
\hline Combinación & $\theta$ & Combinación & $\theta$ \\
\hline $\begin{array}{l}\text { ic_asalud - ic_segsoc } \\
\text { ic_cv - ic_sbv } \\
\text { ic_segsoc - ic_sbv } \\
\text { ic_segsoc-ic_cv } \\
\text { ic_cv - ic_ali } \\
\text { ic_rezedu - ic_sbv } \\
\text { ic_sbv - ic_ali } \\
\text { ic_segsoc - ic_ali }\end{array}$ & $\begin{array}{c}22.542 \\
5.889 \\
4.604 \\
3.493 \\
3.083 \\
2.891 \\
2.742 \\
2.272\end{array}$ & $\begin{array}{l}\text { ic_rezedu - ic_cv } \\
\text { ic_rezedu - ic_ali } \\
\text { ic_rezedu - ic_segsoc } \\
\text { ic_rezedu - ic_asalud } \\
\text { ic_asalud - ic_cv } \\
\text { ic_asalud - ic_sbv } \\
\text { ic_asalud - ic_ali }\end{array}$ & $\begin{array}{l}2.118 \\
1.777 \\
1.460 \\
1.349 \\
1.315 \\
1.181 \\
1.165\end{array}$ \\
\hline
\end{tabular}

Fuente: Elaboración propia en R a partir de MCS-ENIGH 2012.

Un elemento que también debiera de ser tomado en cuenta es la potencial asociación entre las carencias que conforman el mismo espacio de los derechos sociales. De existir asociación entre estas variables se afectan los resultados al calcular su relación con la variable de pobreza por ingresos. Por ello en el 
Cuadro 8 se muestran las razones de posibilidad de las quince combinaciones resultantes de elegir dos a dos las carencias. Los niveles más altos se reportan en las carencias de acceso a servicios de salud y a seguridad social (ic_asalud e ic_segsoc), seguidos de condiciones y servicios básicos en la vivienda (ic_cv e ic_sbv). Respecto del primer caso, esto se debe a cómo se formulan las carencias desde la LGDS; y en el segundo caso se debe a que ambas variables son complementarias en la descripción de una vivienda. Por otro lado, las variables que reportan asociaciones más débiles son el rezago educativo y el acceso a servicios de salud (ic_rezedu e ic_asalud).

Las medidas de asociación para las combinaciones ic_asalud-ic_segsoc y ic_cv-ic_sbv se reportan en el Cuadro 9. Se destaca que el nivel de concordancia en es una asociación positiva muy clara, implicando que sufrir una carencia aumenta la probabilidad de padecer la otra, sobre todo entre ic_asalud y ic_segsoc. El valor de la $D$ de Somers que se reporta es el valor más alto, el cual ocurre cuando el acceso a la seguridad social y los servicios básicos en la vivienda (ic_segsoc e ic_sbv) se establecen como dependientes. La fuerte asociación encontrada entre las carencias invita a analizar cuáles de ellas sería conveniente dejar en el índice de privación.

\begin{tabular}{|c|c|c|c|c|c|c|c|}
\hline Combinación & C & $\boldsymbol{v}$ & $\gamma$ & $\tau_{\mathrm{b}}$ & $\tau_{c}$ & D & $\rho_{S}$ \\
\hline ic_asalud - ic_segsoc & 0.331 & 0.351 & 0.915 & 0.351 & 0.270 & 0.433 & 0.351 \\
\hline ic_cv-ic_sbv & 0.300 & 0.315 & 0.710 & 0.315 & 0.185 & 0.383 & 0.315 \\
\hline
\end{tabular}

Fuente: Elaboración propia en R a partir de MCS-ENIGH 2012.

El siguiente paso es el estudio de la asociación entre la pobreza de ingresos (plb) y el Índice de Privación Social (IPS), ya que la pobreza multidimensional se define a partir de estas dos variables. Los resultados de aplicar las medidas de asociación se reportan en el Cuadro 10. El valor de la $D$ de Somers presentado es el mayor, y resulta de considerar al IPS como dependiente de plb. De la $\mathrm{V}, \tau_{b}$ y $\tau_{c}$ se observa una asociación fuerte entre las variables, lo cual se confirma al considerar las otras medidas. De esta forma, se encuentra una asociación positiva entre la presencia de pobreza de ingresos y el nivel de carencias padecidas.

Cuadro 10. Medidas de asociación entre plb y ips.

\begin{tabular}{|c|c|c|c|}
\hline Medida & Valor & Medida & Valor \\
\hline$\phi$ & 0.392 & $\tau_{b}$ & 0.347 \\
\hline$C$ & 0.365 & $\tau_{c}$ & 0.434 \\
\hline$V$ & 0.392 & $D$ & 0.435 \\
\hline$\gamma$ & 0.536 & $\rho_{s}$ & 0.386 \\
\hline
\end{tabular}

Nota: El valor de la D de Somers que se reporta es el valor más alto, el cual ocurre cuando ips es la variable dependiente.

Fuente: Elaboración propia en R a partir de MCS-ENIGH 2012. 
La información obtenida hasta aquí invita a omitir la carencia del acceso a los servicios de salud, como se hace en el índice de privación social modificado (ips.sal). Al utilizar este índice para definir a los pobres multidimensionales, apenas 491 individuos salen de dicha categoría. La perspectiva multidimensional se fundamenta en que habrá características del individuo que no puedan ser representadas solamente por el ingreso y que por ello deben incluirse otras dimensiones del bienestar en las medidas. Sin embargo, debe entenderse que aquí la omisión de la variable de carencia en los servicios de salud se sugiere debido a su redundancia con la carencia de seguridad social, y no solamente por sus bajos niveles de asociación con las otras variables. En el Cuadro 11 se comparan las frecuencias de los índices de privación social original y modificado (ips e ips.sal); observándose un considerable incremento en el número de individuos con solamente una carencia, aunado a la reducción en el nivel de dos carencias, debido solamente a la redundancia de la carencia.

Cuadro 11. Frecuencias de los niveles de ips.sal.

\begin{tabular}{|c|c|c|c|c|c|c|c|}
\hline Variable & & Frec. Abso- & $(\%)$ & \multicolumn{2}{|c|}{ Variable } & Frec. Absoluta & (\%) \\
\hline \multirow{7}{*}{ ips } & 0 & $53 ’ 230$ & 25.2 & \multirow{7}{*}{ ips.sal } & 0 & $53^{\prime} 721$ & 25.5 \\
\hline & 1 & $52 ' 888$ & 25.1 & & 1 & $71 ' 267$ & 33.8 \\
\hline & 2 & $52^{\prime} 731$ & 25 & & 2 & 44'598 & 21.1 \\
\hline & 3 & $30 ’ 856$ & 14.6 & & 3 & $26^{\prime} 520$ & 12.6 \\
\hline & 4 & $15^{\prime} 171$ & 7.2 & & 4 & $12^{\prime} 014$ & 5.7 \\
\hline & 5 & $5^{\prime} 120$ & 2.4 & & 5 & 2'830 & 1.3 \\
\hline & 6 & 954 & 0.5 & & & & \\
\hline
\end{tabular}

Fuente: Elaboración propia en R a partir de MCS-ENIGH 2012.

En el Cuadro 12 se muestran los valores de las medidas de asociación que resultan de usar la pobreza de ingresos y el IPS modificado (plb e ips.sal), así como las correlaciones de ips.sal con las otras variables. Nótese un incremento en todas las correlaciones, salvo en el caso del acceso a seguridad social (ic_segsoc). Ante la eliminación de la carencia de servicios de salud, la correlación entre el nuevo IPS y el ingreso se incrementa, sobre todo para el ingreso transformado (ing.bc). Al obtener las medidas de asociación, en todos los casos se encuentra un valor mayor, mostrando una mayor relación entre el espacio de los ingresos y el espacio de los derechos sociales. 
Cuadro 12. Medidas de asociación y correlación usando ips.sal.

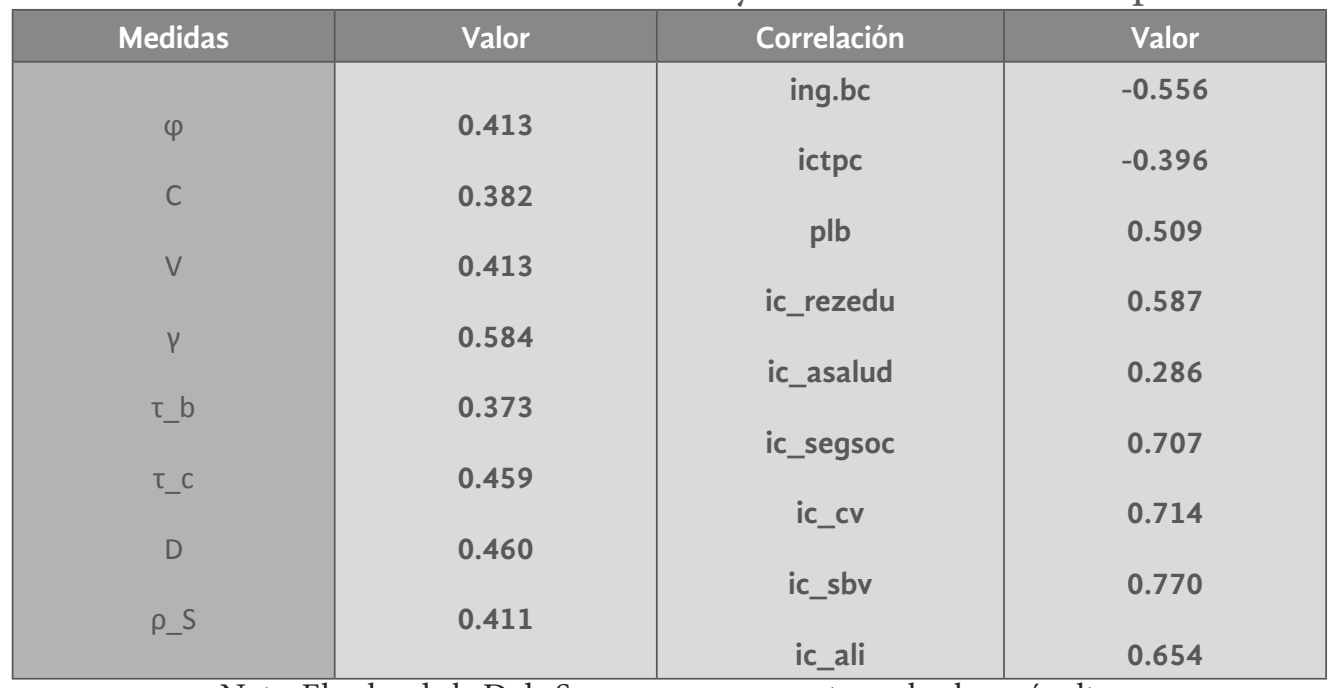

Nota: El valor de la D de Somers que se reporta es el valor más alto, el cual ocurre cuando ips es la variable dependiente.

Fuente: Elaboración propia en R a partir de MCS-ENIGH 2012.

Como un ejercicio final, se comparan las medidas de CONEVAL usando el IPS original y el modificado. Los resultados se ofrecen en el Cuadro 13, para lo cual se sugiere revisar Alkire y Foster (2011) y Sáenz et al. (2015) a fin de facilitar la interpretación ${ }^{16}$. En términos de la proporción de pobres $(H)$ el cambio es apenas notorio; no obstante, el número de carencias promedio padecidas por los pobres multidimensionales $\left(\mathrm{C}_{\text {prom }}\right)$ empieza a generar diferencias. El índice A representa la proporción de carencias padecidas por los pobres multidimensionales, mientras el $M_{0}$ señala la proporción de carencias sociales padecidas por la población total, donde la redundancia referente a los servicios de salud genera cambios importantes en la estimación de la medida.

Cuadro 13. Medidas de pobreza usando ips e ips.sal.

\begin{tabular}{|c|c|c|}
\hline Medidas & ips & ips.sal \\
\hline$H$ & 0.4678 & 0.4671 \\
$C_{\text {prom }}$ & 2.376 & 2.138 \\
$A$ & 0.396 & 0.356 \\
$M_{0}$ & 0.185 & 0.166 \\
\hline
\end{tabular}

Fuente: Elaboración propia en R a partir de MCS-ENIGH 2012.

Los resultados que se han mostrado hasta el momento indican la existencia de asociación entre el espacio del bienestar y el de los derechos sociales. Las variables que representan estos espacios mues-

16- En cuanto al IPS original los valores cambian ligeramente de los ofrecidos por el Consejo en su página oficial ya que aquí se está utilizando una muestra menor a la original. 
tran niveles de asociación considerables, incluso mayores cuando se sugiere la omisión de la carencia de acceso a servicios de salud en el estudio. Dado que el acceso a este derecho se establece en la LGDS, es necesario ofrecer alternativas, de manera que no sea necesario omitir la carencia. Una opción es buscar alguna ponderación que permita seguir considerándola en el espacio de los derechos sociales. O bien, sugerir otra forma de medir el acceso a los servicios de salud, a partir de la información recabada por el mismo MCS de la ENIGH.

\section{Conclusión}

La definición de pobreza multidimensional requiere que el individuo esté privado en el espacio del bienestar y en el de los derechos sociales. En términos estadísticos, la independencia de estos eventos implicaría que la ocurrencia de un evento no se ve afectada por la ocurrencia del otro. Para el CONEVAL, la condición de ser pobre de ingresos no se ve afectada por la presencia de carencias y viceversa; aunque dicho supuesto teórico no se confirma a través de un examen estadístico.

En el presente estudio se cumple el objetivo de mostrar que, empíricamente, existe asociación entre el ingreso y los derechos sociales, más allá de los supuestos teóricos. Dicha asociación representa un reto para la política pública en México, ya que de los seis derechos considerados, solamente el acceso a servicios de salud logró un bajo nivel de dependencia. Este resultado no es contradictorio a lo que estudios como el de Battiston et al. (2013) y CEPAL (2013:78), donde se destaca que el ingreso para países en desarrollo sigue siendo una dimensión de la pobreza de considerable importancia. Aunado a lo anterior debe señalarse que dado el nivel de asociación encontrado entre el ingreso y las carencias, sería viable considerarlo dentro de la medida de profundidad de la pobreza como una dimensión más, ya que los resultados invitan a pensar que sigue siendo un factor determinante en el acceso a ciertos derechos sociales.

De los resultados obtenidos en el análisis de asociación se destacan algunas conclusiones. Primero, existe asociación entre el espacio del bienestar y el espacio de los derechos sociales. Se mostró mediante diferentes pruebas que existe una relación entre la condición de ser pobre de ingresos y la de padecer carencias; todas las pruebas fueron estadísticamente significativas. Las variables de los derechos que mostraron mayor asociación con el ingreso son el acceso a la seguridad social y las relacionadas con la vivienda. Una conjetura es que la seguridad social representa, en cierta manera, el acceso a un empleo formal y mejor remunerado. Mientras que las características de la vivienda para ser mejoradas requieren de un incremento sustancial del ingreso, por lo que su asociación es alta en comparación a otras variables.

También se encontró asociación entre las variables que conforman al espacio de los derechos sociales. Las carencias de acceso a servicios de salud y acceso a la seguridad social presentan la mayor asociación, seguidas de las carencias en condiciones de la vivienda y en servicios básicos de la vivienda. Respecto de las primeras, su fuerte relación se debe a la forma en que se definieron en la LGDS. En cuanto a las variables referentes a la vivienda, si bien consideran distintos elementos, ambas pertenecen a una misma dimensión. Ello sugiere una forma de medición alternativa dentro del espacio de los derechos, de manera que no haya una doble ponderación a una misma dimensión.

La carencia en el acceso a los servicios de salud también mostró resultados importantes. Por un lado, la relación entre la pobreza de ingresos y esta carencia es mínima, pero existe y puede ser detectada a través 
de las diferentes medidas. No obstante, la asociación entre el espacio del bienestar y el de los derechos resulta más notoria cuando se omite del IPS. La sugerencia que se desprende es la de proponer un IPS ponderado de una manera distinta; tal que sin eliminar la variable ic_asalud, ésta pueda integrarse con ic_segsoc. Lo anterior forma parte de una investigación posterior, en la que se re-ponderen estas dos dimensiones, y ello resulte en un nuevo IPS que permita mejorar las estimaciones de la pobreza multidimensional.

No se propone que se vuelva a la medición tradicional de la pobreza, desde el enfoque monetario. Se sugiere que se analice la posibilidad de agregar al ingreso y de corregir la redundancia en lo referente a los servicios de salud. Efectivamente, cambiar el formato de medición, o el peso de las dimensiones, generará cambios en la estimación de la pobreza, pero los resultados serán robustos en términos de la ordenación de los individuos pobres, como lo muestra el ejercicio realizado en Alkire y Santos (2014).

Como se señala en Sáenz et al. (2015) el formato con el cual actualmente se elabora la medición de pobreza no genera un problema en sí mismo. Es decir, los derechos sociales se evalúan como dicotomías (tiene, o no, el acceso al derecho) y no como un nivel de carencia; por ello que solamente sea posible obtener la medida $M_{0}$. No obstante, el problema radica en la imposibilidad de elaborar medidas sobre la profundidad y severidad de la pobreza, las cuales brindarían información sobre el cambio en los niveles de pobreza en el tiempo, fruto de las transferencias y los programas de política pública, sobre todo si se incluyera al ingreso como una dimensión adicional. El otro inconveniente que presenta la medición actual es la redundancia entre los derechos sociales, en particular, entre el acceso a servicios de salud y el acceso a seguridad social, dicha redundancia es clara desde la definición en la LGDS y se corrobora estadísticamente en este ejercicio. En cuanto a sus consecuencias, el hecho de que cualquier persona sea carente en uno de esos derechos implica que prácticamente lo será de forma simultánea en el otro, provocando la sobre-estimación del nivel de carencias, lo que a su vez tiene un efecto inmediato en la estimación de la medida $M_{0}$.

\section{Referencias}

Agresti, Alan. 2002. Categorical data analysis. New Jersey, Estados Unidos: Wiley-Interscience. Alkire, Sabina y James Foster. 2009. Memo to CONEVAL. www.ophi.org.uk/wp-content/uploads/OPHIRP-21a.pdf (18 de abril de 2016).

Alkire, Sabina y James Foster. 2011. Counting and multidimensional poverty measurement. Journal of Public Economics, 95(7): 476-487. DOI: https://doi.org/10.1016/j.jpubeco.2010.11.006

Alkire, Sabina y Maria Emma Santos. 2014. Measuring acute poverty in the developing world: Robustness and scope of the multidimensional poverty index. World Development, 59: 251-274. DOI: https:// doi.org/10.1016/j.worlddev.2014.01.026

Alkire, Sabina, James Foster, Suman Seth, Maria Emma Santos, José Manuel Roche y Paola Ballón. 2015. Multidimensional poverty measurement and analysis. Estados Unidos: Oxford University Press.

Amores, César. 2014. Medición de la pobreza multidimensional: El caso ecuatoriano. www.ophi.org.uk/ medicion-de-la-pobreza-multidimensional-el-caso-ecuatoriano/ (12 de septiembre de 2016).

Banco Mundial. 2000. The nature and evolution of poverty. En: World Development Report 2000/2001 Attacking Poverty. Nueva York: Oxford University Press, 15-29.

Battiston, Diego, Guillermo Cruces, Luis López-Calva, Maria Ana Lugo y Maria Emma Santos. 2013. Income and beyond: Multidimensional poverty in six Latin American countries. Social Indicators Research, 112(2): 291:314. 
Botsch, Bob. 2011. Significance and measures of association, Scopes and methods of political science. http://polisci.usca.edu/apls301/Text/Chapter\%2012.\%20Significance\%20and\%20Measures\%20of\%20 Association.htm (18 de abril de 2016).

Bourguignon, Francois y Satya Chakravarty. 2003. The measurement of multidimensional poverty. Journal of Economic Inequality, 1(1): 25-49. DOI: https:/doi.org/10.1023/A:1023913831342

CEPAL. 2013. Avances recientes en la reducción de la pobreza. En: Panorama Social de América Latina. Santiago de Chile: CEPAL, 55-87.

CONEVAL. 2009. Metodología para la medición multidimensional de la pobreza en México. México: CONEVAL.

Foster, James. 2010. Informe sobre la medición multidimensional. En Medición multidimensional de la pobreza en México, editado por Minor Mora. México: El Colegio de México, CONEVAL, 323:399.

Kirk, David. 1973. On the numerical approximation of the bivariate normal (tetrachoric) correlation coefficient. Psychometrika, 38 (2): 259-268. DOI: https://doi.org/10.1007/BF02291118

Martí, Arturo, Guiomar Peña, Sergio Muñoz, Gabriela Comunián y Arturo Martí. 2006. Significado de la razón de posibilidades (Odds Ratio). Gaceta Médica de Caracas, 114 (1): 13-16.

McKenzie, David. 2005. Measuring inequality with asset indicators. Journal of Population Economics, 18 (2): 229-260. DOI: https://doi.org/10.1007/s00148-005-0224-7

Nolan, Brian y Christopher Whelan. 1996. The relationship between income and deprivation: A dynamic perspective. Revue économique, 47 (3): 709-717. DOI: https://doi.org/10.2307/3502573

Olsson, Ulf, Fritz Drasgow y Neil Dorans. 1982. The polyserial correlation coefficient. Psychometrika, 47 (3): 337-347. DOI: https://doi.org/10.1007/BF02294164

Ram, Rati. 1982. Composite indices of physical quality of life, basic needs fulfillment, and income. A principal component representation. Journal of Development Economics, 11 (2): 227-247. DOI: https:// doi.org/10.1016/0304-3878(82)90005-0

Sáenz, Hada. 2016. Revisión y evaluación a la medición de la pobreza multidimensional en México. Tesis de doctorado, Saltillo, Coahuila: Centro de Investigaciones Socioeconómicas.

Sáenz, Hada, Luis Gutiérrez y Enrique Minor. 2015. Aproximación para analizar el índice de intensidad de la pobreza multidimensional de México. Iztapalapa, Revista de Ciencias Sociales y Humanidades, 36 (79): 151-169.

Sen, Amartya. 1983. Poor, relatively speaking. Oxford Economic Papers, 35 (2):153-169.

Sen, Amartya. 1992. Sobre conceptos y medidas de la pobreza. Comercio Exterior, 42(4): 310-322.

Sheskin, David. 2004. Handbook of parametric and nonparametric statistical procedures. Estados Unidos: Chapman and Hall CRC.

Tapia, José y Javier Nieto. 1993. Razón de posibilidades: Una propuesta de traducción de la expresión Odds Ratio. Salud Pública de México, 35 (4): 419-424.

Ucar, Baris. 2015. The usability of asset index as an indicator of household economic status in Turkey: Comparison with expenditure and income data. Social Indicators Research, 121 (3): 745-760. DOI: https://doi.org/10.1007/s11205-014-0670-2

Visauta, Bienvenido. 2007. Análisis estadístico con SPSS 14: Estadística básica. Madrid: McGraw-Hill. $\mathrm{Yu}$, Jiantuo. 2013. Multidimensional poverty in China: Findings based on the CHNS. Social Indicators Research, 112(2), pp. 315-336. DOI: https://doi.org/10.1007/s11205-013-0250-x 
- Revista de Ciencias Sociales y Humanidades. ISSN-P: 0188-9834 ISSN-E: 2395-8669.

\section{Anexo}

Cuadro A. Definición de las carencias en el espacio de los derechos sociales.

\begin{tabular}{|c|c|}
\hline Carencia & Definición \\
\hline Rezago Educativo & $\begin{array}{l}\text { Una persona se encuentra en situación de rezago educativo si cumple alguno de los } \\
\text { siguientes criterios: } \\
\text { Tiene de tres a quince años, no cuenta con la educación básica obligatoria y no asiste } \\
\text { a un centro de educación formal. } \\
\text { Nació antes de } 1982 \text { y no cuenta con el nivel de educación obligatoria vigente en el } \\
\text { momento en que debía haberla cursado (primaria completa). } \\
\text { Nació a partir de } 1982 \text { y no cuenta con el nivel de educación obligatoria (secundaria } \\
\text { completa). }\end{array}$ \\
\hline $\begin{array}{c}\text { Acceso a servicios de } \\
\text { salud }\end{array}$ & $\begin{array}{l}\text { Una persona se encuentra en situación de carencia por acceso a los servicios de salud } \\
\text { cuando: } \\
\text { No cuenta con adscripción o derecho a recibir servicios médicos de alguna institución } \\
\text { que los presta, incluyendo el Seguro Popular, las instituciones públicas de seguridad } \\
\text { social (IMSS, ISSSTE federal o estatal, Pemex, Ejército o Marina) o los servicios médi- } \\
\text { cos privados. }\end{array}$ \\
\hline $\begin{array}{c}\text { Acceso a la seguridad } \\
\text { social }\end{array}$ & $\begin{array}{l}\text { Se identifica a la población con carencia por acceso a la seguridad social de acuerdo } \\
\text { con los siguientes criterios: } \\
\text { En cuanto a la población económicamente activa, asalariada, se considera que no } \\
\text { tiene carencia en esta dimensión si disfruta, por parte de su trabajo, de las prestacio- } \\
\text { nes establecidas en el artículo } 2^{\circ} \text { de la Ley del Seguro Social (o sus equivalentes en las } \\
\text { legislaciones aplicables al apartado B del Artículo } 123 \text { constitucional). } \\
\text { Dado el carácter voluntario de la inscripción al sistema por parte de ciertas categorías } \\
\text { ocupacionales, en el caso de la población trabajadora no asalariada o independiente se } \\
\text { considera que tiene acceso a la seguridad social cuando dispone de servicios médicos } \\
\text { como prestación laboral o por contratación voluntaria al régimen obligatorio del } \\
\text { Instituto Mexicano del Seguro Social y, además, cuenta con Sistema de Ahorro para el } \\
\text { Retiro o Administradoras de Fondos para el Retiro. } \\
\text { Para la población en general, se considera que tiene acceso cuando goce de alguna } \\
\text { jubilación o pensión, o sea familiar de una persona dentro o fuera del hogar con acceso } \\
\text { a la seguridad social. } \\
\text { En el caso de la población en edad de jubilación (sesenta y cinco años o más), se } \\
\text { considera que tiene acceso a la seguridad social si es beneficiario de algún programa } \\
\text { social de pensiones para adultos mayores. } \\
\text { La población que no cumpla con alguno de los criterios mencionados, se considera en } \\
\text { situación de carencia por acceso a la seguridad social. }\end{array}$ \\
\hline $\begin{array}{c}\text { Calidad y espacios de la } \\
\text { vivienda }\end{array}$ & $\begin{array}{l}\text { Se considera como población en situación de carencia por calidad y espacios de la } \\
\text { vivienda a las personas que residan en viviendas que presenten, al menos, una de las } \\
\text { siguientes características: } \\
\text { El material de los pisos de la vivienda es de tierra. } \\
\text { El material del techo de la vivienda es de lámina de cartón o desechos. } \\
\text { El material de los muros de la vivienda es de embarro o bajareque; de carrizo, bambú o } \\
\text { palma; de lámina de cartón, metálica o asbesto; o material de desecho. } \\
\text { La razón de personas por cuarto (hacinamiento) es mayor que } 2.5 \text {. }\end{array}$ \\
\hline
\end{tabular}


- Revista de Ciencias Sociales y Humanidades. ISSN-P: 0188-9834 ISSN-E: 2395-8669.

\begin{tabular}{|c|c|}
\hline Carencia & Definición \\
\hline $\begin{array}{l}\text { Acceso a los servicios } \\
\text { básicos en la vivienda }\end{array}$ & $\begin{array}{c}\text { Se considera como población en situación de carencia por servicios básicos en la } \\
\text { vivienda a las personas que residan en viviendas que presenten, al menos, una de las } \\
\text { siguientes características: } \\
\text { El agua se obtiene de un pozo, río, lago, arroyo, pipa; o bien, el agua entubada la obtie- } \\
\text { nen por acarreo de otra vivienda, o de la llave pública o hidrante. } \\
\text { No cuentan con servicio de drenaje, o el desagüe tiene conexión a una tubería que va } \\
\text { a dar a un río, lago, mar, barranca o grieta. } \\
\text { No disponen de energía eléctrica. } \\
\text { El combustible que se usa para cocinar o calentar los alimentos es leña o carbón sin } \\
\text { chimenea. }\end{array}$ \\
\hline Acceso a la alimentación & $\begin{array}{l}\text { Se considera en situación de carencia por acceso a la alimentación a los hogares que: } \\
\text { Presenten un grado de inseguridad alimentaria moderado o severo }\end{array}$ \\
\hline
\end{tabular}

Fuente: Coneval (2009) 